\title{
Wie vollständig ist der Bundeseinheitliche Medikationsplan? Eine Analyse bei Krankenhausaufnahme
}

\section{How complete is the Germany-wide standardised medication list (“Bundeseinheitlicher Medikationsplan")? An analysis at hospital admission.}

Autoren

Stefanie Amelung1, 2, 3, Bianca Bender ${ }^{1}$, Andreas Meid ${ }^{3}$, Stefanie Walk-Fritz, ${ }^{1}$, Torsten Hoppe-Tichy ${ }^{1,2}$, Walter E. Haefeli2, ${ }^{(0)}$, Hanna M. Seidling ${ }^{2,3}$

\section{(c) $(1) \ominus$}

Institute

1 Apotheke des Universitätsklinikums Heidelberg, Heidelberg, Deutschland

2 Kooperationseinheit Klinische Pharmazie, Universitätsklinikum Heidelberg, Heidelberg, Deutschland

3 Abteilung Klinische Pharmakologie und Pharmakoepidemiologie, Universitätsklinikum Heidelberg, Heidelberg, Deutschland

Schlüsselwörter

Arzneimitteltherapiesicherheit, Arzneimittelanamnese, Bundeseinheitlicher Medikationsplan, Diskrepanzen

Key words

patient safety, medication reconciliation, standardised medication list, information discrepancies

Bibliografie

Dtsch Med Wochenschr 2020; 145: e116-e122

Online-Publikation: 6.10.2020

DOI $10.1055 / \mathrm{a}-1212-2836$

ISSN 0012-0472

(c) 2020. The Author(s).

This is an open access article published by Thieme under the terms of the Creative Commons Attribution-NonDerivative-NonCommercial License, permitting copying and reproduction so long as the original work is given appropriate credit. Contents may not be used for commecial purposes, or adapted, remixed, transformed or built upon. (https://creativecommons.org/licenses/by-nc-nd/4.0/)

Korrespondenzadresse

PD Dr. sc. hum. Hanna Seidling

Kooperationseinheit Klinische Pharmazie

Abteilung Klinische Pharmakologie und

Pharmakoepidemiologie

Universitätsklinikum Heidelberg, Im Neuenheimer Feld 410,

69120 Heidelberg, Deutschland

Hanna.Seidling@med.uni-heidelberg.de

\section{ZUSAMMENFASSUNG}

Einleitung Bei stationärer Aufnahme scheint die Aktualität und Vollständigkeit des Bundeseinheitlichen Medikationsplans häufig nicht gegeben. Ebenso ist unklar, welche Charak- teristiken der Pläne die Wahrscheinlichkeit für Diskrepanzen erhöhen.

Methoden Retrospektiv wurden deshalb 100 Pläne, die zur Arzneimittelanamnese elektiver Patienten einer chirurgischen Klinik mitgebracht wurden, geprüft, ob und welche Abweichungen bestanden. Die Abweichungen wurden 7 Kategorien zugeordnet: Arzneimittel, das in der Anamnese erfasst wurde, fehlt auf dem Plan, Arzneimittel auf dem Plan wird nicht mehr eingenommen, Stärke oder Dosierung fehlt auf dem Plan bzw. ist falsch oder die Darreichungsform ist falsch dokumentiert. Hinweise zur Arzneimitteltherapiesicherheit, involvierte Arzneimittel und -formen wurden ebenfalls erfasst. Mithilfe multivariater Analysen wurde der Einfluss der Aktualität, der Anzahl der Arzneimittel und der ausstellenden Facharztdisziplin der Pläne auf die Art und Anzahl an Diskrepanzen untersucht.

Ergebnisse Zur Arzneimittelanamnese wiesen $78 \%$ (78/100) der Pläne Abweichungen auf. Insgesamt wurden 226 Abweichungen (2,3 $\pm 0,6$ Abweichungen/Anamnese) dokumentiert. Am häufigsten fehlte ein Arzneimittel auf dem Plan ( $n=103)$. Von allen Hinweisen und Empfehlungen betrafen 64\% (83/177) das perioperative Management von Antithrombotika $(n=55)$ und Antidiabetika $(\mathrm{n}=28)$. In der multivariaten Analyse stieg nur das Risiko für fehlerhafte Angaben bei Stärke und Dosierung mit dem Alter der Pläne signifikant $(p=0,047)$ und war um mehr als das 2-fache erhöht, wenn der Plan älter als einen Monat war. Diskussion Die Aktualität, Vollständigkeit und Aspekte der Arzneimitteltherapiesicherheit des Bundeseinheitlichen Medikationsplans sollten umfassend und gezielt im Anamnesegespräch validiert werden. In der Praxis sollten Pläne, die älter als 1 Monat sind, besonders kritisch hinsichtlich Angaben zu Stärke und Dosierung geprüft und der Plan entsprechend regelmäßig aktualisiert werden.

\section{ABSTRACT}

Background At inpatient admission, the timeliness and completeness of the Germany-wide standardised medication list ("Bundeseinheitlicher Medikationsplan") often seems inappropriate. It is also unclear which characteristics of the lists increase the probability of discrepancies. 
Methods A total of 100 medication lists of elective patients of a surgical clinic were retrospectively evaluated with regard to potential discrepancies compared to the standardised medication reconciliation. The discprepancies were assigned to 7 categories: drug taken is missing on the list, drug on the list is no longer taken, strength or dosage is missing at the list or is incorrect, or the documented dosage form is different. Advice on patient safety, involved drugs and dosage forms were also recorded. Multivariate analyses were used to investigate the influence of the timeliness, number of drugs and issuing medical specialty of the lists on the type and number of discrepancies.

Results Compared to the medication reconciliation, $78 \%$ (78/100) of the lists showed discrepancies. A total of 226 deviations ( $2.3 \pm 0.6$ deviations/list) were documented. Most of- ten, a drug was missing from the list $(n=103)$. Of all recommendations, $64 \%(83 / 177)$ concerned the perioperative management of anticoagulants $(n=55)$ and antidiabetics $(n=28)$, corresponding to $62 \%(62 / 100)$ of the lists. In the multivariate analysis, only the risk of incorrect information on strength and dosage increased significantly with the age of the lists $(p=0.047)$ and was more than twice as high when the list was more than one month old.

Conclusions The timeliness, completeness and aspects of patient safety must be comprehensively validated. Medication lists that are older than 1 month should be checked particularly critically with regard to information on strength and dosage and the plan should be updated accordingly at regular intervals.

\section{Einleitung}

Abweichungen zwischen der Aufnahmemedikation im Krankenhaus und der jeweiligen Vormedikation eines Patienten sind aufgrund einer unvollständig erhobenen Arzneimittelanamnese (AM-Anamnese) in 2 von 3 Fällen zu finden, wobei je nach Studie 11-59\% der Diskrepanzen als klinisch relevant erachtet werden [1]. Um die Arzneimitteltherapiesicherheit zu erhöhen, ist eine qualitativ hochwertige AM-Anamnese Voraussetzung für eine ebenso hochwertige stationäre Weiterführung der Therapie und auch für ein qualitativ hochwertiges Entlassmanagement. Denn immerhin $40 \%$ der Medikationsfehler in der Aufnahmemedikation werden in ärztlichen Entlassbriefen wiedergefunden [2], wobei mindestens ein Fünftel dieser Abweichungen als potenziell kritisch für den Patienten bewertet werden [3-5].

Um die Arzneimitteltherapiesicherheit unter anderem an der Schnittstelle ambulante-stationäre Versorgung zu verbessern, wurde zum 1. Oktober 2016 im Rahmen des E-Health-Gesetzes die Einführung des Bundeseinheitlichen Medikationsplans (BMP) beschlossen (§31a SGB V). Der BMP soll dabei möglichst alle verschreibungspflichtigen Arzneimittel sowie relevante Selbstmedikation enthalten, wobei die gesamte Information des Plans über den auf dem Plan aufgedruckten Data-Matrix-Code digital in zertifizierte Softwaresysteme eingelesen, aktualisiert und als neuer Plan wieder ausgedruckt werden kann [6].

Prinzipiell hat der BMP daher das Potenzial, eine schnell zugängliche Informationsquelle auch für die AM-Anamnese bei der stationären Aufnahme zu sein, die das Anamnesegespräch nicht nur strukturieren, sondern, ggf. auch unter Einsatz technischer Hilfsmittel wie Data-Matrix-Code-Scanner, verkürzen könnte. Voraussetzung hierfür ist jedoch eine im Sinne der Vollständigkeit und Aktualität hohe Qualität der auf dem BMP dokumentierten Informationen.

Es sollte deshalb systematisch untersucht werden, wie häufig ein BMP in der AM-Anamnese vorhanden ist und ob es Abweichungen zwischen den Angaben des BMP und der strukturierten AMAnamnese bei Krankenhausaufnahme gibt. Aus den Ergebnissen soll abgeleitet werden, auf welche möglichen Diskrepanzen bei der AM-Anamnese besonders geachtet werden muss und wie diese bei der Erstellung eines BMP möglichst verhindert werden könnten.

\section{Methoden}

Über einen Zeitraum von 6 Monaten (10/2017-03/2018) wurden retrospektiv und pseudonymisiert für alle pharmazeutischen AM-Anamnesen der erwachsenen Patienten der Chirurgischen Universitätsklinik Heidelberg alle verfügbaren Informationsquellen dokumentiert. Die pharmazeutische AM-Anamnese erfolgte standardisiert und qualitätsgesichert basierend auf einer Checkliste, welche die Empfehlungen des High-5s-Projektes zu Medication Reconciliation aufgreift [7]: Apotheker*innen der Klinikapotheke des Universitätsklinikums Heidelberg erhoben anhand von mindestens 2 unterschiedlichen Quellen (z. B. Patienten-/Angehörigengespräch und Medikationspläne, mitgebrachte Arzneimittelpackungen, Rückfragen an verordnende Arztpraxis etc.) systematisch eine möglichst vollständige Liste der aktuellen bzw. in letzter Zeit eingenommenen Arzneimittel des Patienten (die sogenannte „Eintrittsmedikation“). Ergaben sich Abweichungen zwischen der bisherigen Verordnung und der Medikation, wie der Patient sie zuletzt eingenommenen hat, wurde dies für die weiterbehandelnden Ärzte auf Station dokumentiert. Die Medikation wurde anschließend auf arzneimittelbezogene Probleme (ABP) hin überprüft und auf die im Krankenhaus gelisteten Arzneimittel umgestellt (die sogenannte „Klinikmedikation“). Bei der ABP-Prüfung wurde insbesondere auf das perioperative Management bestimmter Arzneimittel geachtet. Die AM-Anamnese wurde zunächst im Gespräch bzw. papierbasiert durchgeführt, anschließend erfolgte die Eingabe der Eintrittsmedikation in die Software AiDKlinik ${ }^{\circledR}$, wo auch die Umstellung auf die Klinikmedikation durchgeführt wurde. Eintrittsmedikation, die vorgeschlagene Klinikmedikation und etwaige Anmerkungen, Empfehlungen und Hinweise aus der ABP-Prüfung wurden anschließend anhand des sogenannten „Switch-Ausdrucks“ (ein von der Software erstelltes pdf-Dokument mit den erfassten Informationen und einem qualifizierten Vorschlag zur Umstellung auf die Hausliste) bei Bedarf mit Ärzten oder Pflegekräften besprochen. 


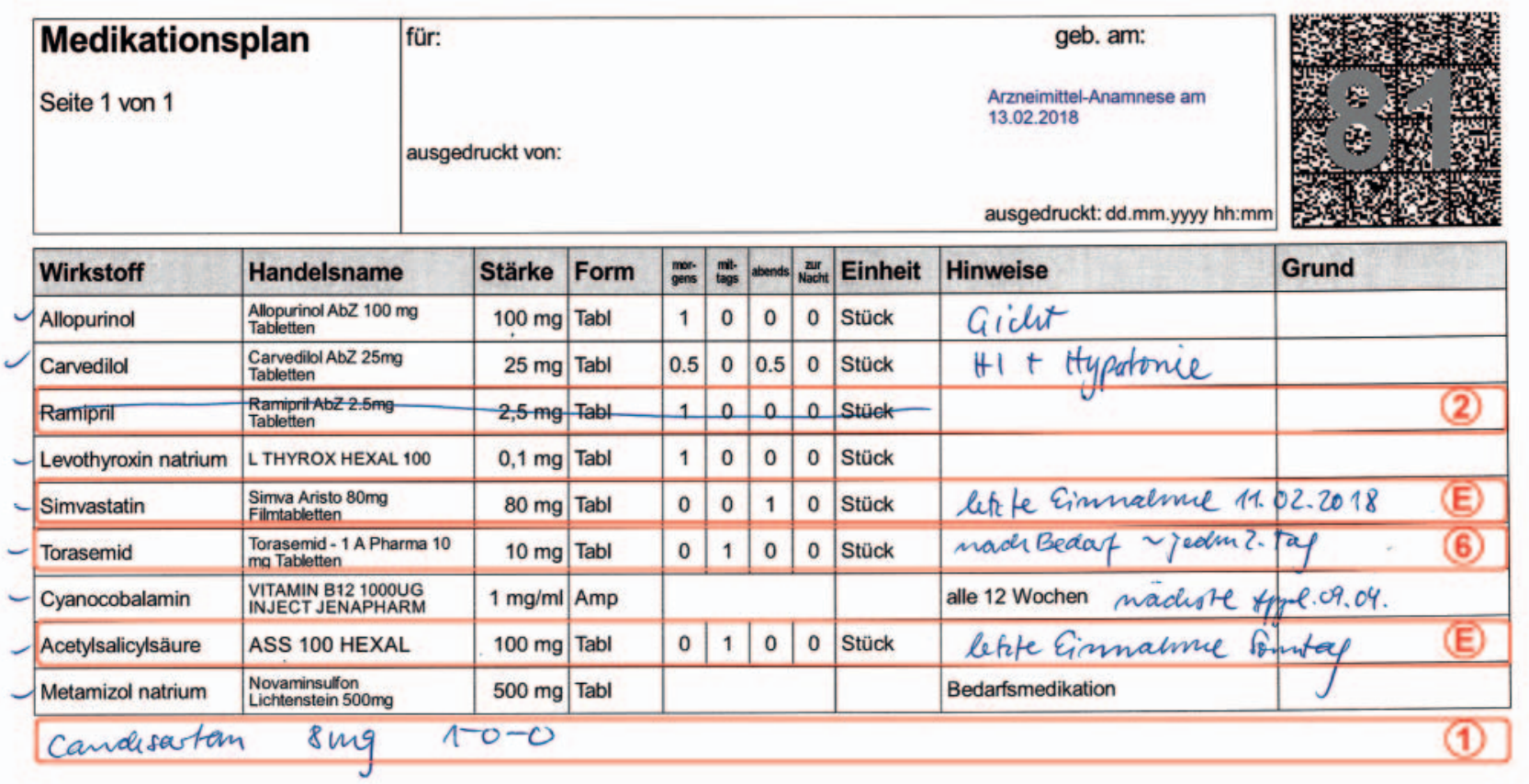

- Abb. 1 Beispiel einer fiktiven Diskrepanzanalyse mit Notizen der Apothekerin (blau) und den gefundenen Diskrepanzen (rot). Im Rahmen der Arzneimittelanamnese stellt sich heraus, dass Ramipril nicht mehr eingenommen wird (2), Arzneimittel wird nicht mehr eingenommen), dafür aber Candesartan (1), Arzneimittel fehlt), und Torasemid nur nach Bedarf eingenommen wird (6), falsche Dosierung). Im Rahmen des perioperativen Managements wird u. a. die letzte Einnahme des Blutverdünners ASS erfasst (ㅌ), pharmazeutische Empfehlung/Hinweis). Neben den erhobenen Eigenschaften Alter des BMP, ausstellende Facharztdisziplin und verwendete Software wurden die erhobenen Präparate gezählt und in rezeptpflichtig und rezeptfrei eingeteilt. dd.mm.yyy hh:mm... Datum und Uhrzeit.

Anhand aller im Zeitraum dokumentierten AM-Anamnesen wurde der Anteil an mitgebrachten BMP zur AM-Anamnese berechnet. Anschließend wurden 100 konsekutiv ausgewählte BMP genauer untersucht. Als BMP-Eigenschaften wurden das Alter des BMP (als Differenz des BMP-Druckdatums zum Datum der AM-Anamnese), die ausstellende Facharztdisziplin, die verwendete Software, aus der der BMP gedruckt wurde, und die Anzahl der aufgelisteten Präparate, unterteilt in rezeptpflichtig und rezeptfrei, dokumentiert.

Der Inhalt des BMP wurde mit der entsprechenden Eintrittsmedikation verglichen, wobei die Eintrittsmedikation als Goldstandard definiert wurde. Angelehnt an Steuerbaut et al. wurden alle Unstimmigkeiten folgenden 7 Diskrepanzenarten zugeordnet [8]: Arzneimittel, das in der AM-Anamnese erfasst wurde, fehlt auf dem BMP (1), Arzneimittel auf dem BMP wird nicht mehr eingenommen (2), Stärke (3) oder Dosierung fehlt auf dem BMP (4), oder Stärke (5) oder Dosierung auf dem BMP ist falsch (6), oder die Darreichungsform auf dem BMP ist falsch (7). Ebenso wurden pharmazeutische Empfehlungen oder Hinweise der ABP-Prüfung an die behandelnden Ärzte (E) erfasst. Die Analyse erfolgte im 4-Augen-Prinzip. Zudem wurden die involvierten Präparate gemäß Wirkstoff/-gruppe (entsprechend ihres Level-1-ATC-Codes, anatomisch-therapeutisch-chemisches Klassifikationssystem) und Arzneiform gruppiert (siehe auch $>$ Abb. 1).

Um Assoziationen zwischen den 7 definierten Diskrepanzen und den BMP-Eigenschaften als Einflussvariablen zu untersuchen, wurden die Diskrepanzen als Zielgrößen festgelegt, als Anzahl an Diskrepanzen operationalisiert und separate verallgemeinerte lineare Modelle auf Basis der negativen Binomialverteilung mit Log-Linkfunktion erstellt. Das Risiko der einzelnen BMP-Eigenschaften auf die Art der Diskrepanzen wurde als Inzidenzratenverhältnis multivariat beschrieben. Darauf aufbauend wurde untersucht, ob sich ein Alter des BMP in Tagen definieren lässt, ab dem das Risiko für Diskrepanzen steigt. Dazu wurde auf Basis desselben Modells univariat eine optimale Trenngrenze in der Verteilung des BMP-Alters über den sogenannten Minimum-p-Value-Ansatz ermittelt [9]; als optimale Trenngrenze gilt demnach dasjenige Alter des BMPs, welches bei Überschreitung das Risiko im Verhältnis zu den Tagen unterhalb der Trenngrenze gemessen am kleinsten $\mathrm{p}$-Wert maximiert.

Die statistischen Analysen wurden mit R Software/Environment Version 3.5.1 ( $R$ foundation for statistical computing, Wien, Österreich) durchgeführt. Die Aufbereitung und Analyse der Daten erfolgten mithilfe MS Excel 2010 (Microsoft, Redmond, USA). Die kategorischen Variablen wurden als Häufigkeit und in Prozent angegeben.

Das Studienprotokoll wurde von der zuständigen Ethikkommission der Medizinischen Fakultät der Universität Heidelberg genehmigt (Nummer des Ethikvotums: S-454/2019). 


\section{Diskrepanzen (1) - (7) \\ (1) Arzneimittel fehlt}

(2) Arzneimittel nicht mehr eingenommen

(3) Stärke fehlt

(4) Dosierung fehlt

(5) Stärke falsch

(6) Dosierung falsch

(7) Andere Darreichungsform

Diskrepanzen (1) - (7) + Empfehlungen

pharmazeutische Empfehlungen

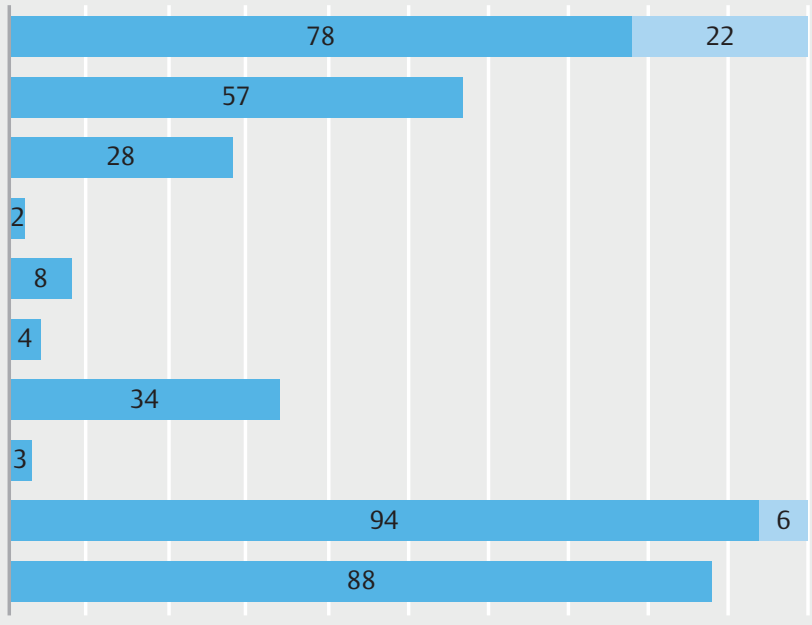

- Abb.2 Anteil betroffener BMP nach Art der Diskrepanzen in 100 BMP. Dunkelblau: Anteil BMP mit Diskrepanzen, Mehrfachzählung pro BMP möglich. Hellblau: Anteil BMP ohne Diskrepanzen.

\section{Ergebnisse}

\section{Anteil des BMP in der Arzneimittel-Anamnese und seine Eigenschaften}

Insgesamt wurden im untersuchten Zeitraum 931 AM-Anamnesen verzeichnet; darunter waren für 271 Patienten keine oder weniger als 3 systemisch wirksame Dauermedikamente dokumentiert. Für 142 AM-Anamnesen brachten Patienten einen BMP mit. Dies entspricht einem BMP-Anteil von 15,3\% (142/931) aller AMAnamnesen bzw. 21,5\% (142/660) der Patienten mit mindestens 3 systemisch wirksamen Dauermedikamenten.

Die 100 exemplarisch untersuchten BMP waren im Mittel 44 Tage alt (Median = 13 Tage, q1 =6, q3 = 43) und wurden in Praxen von Fachärzten für Allgemeinmedizin $(n=59)$, Innere Medizin $(n=34)$ oder Fachärzten für Innere und Allgemeinmedizin $(n=2)$ ausgestellt. Fünf BMP wurden am Universitätsklinikum Heidelberg ausgestellt, wobei Informationen zu Autor und Fachabteilung nicht auf dem BMP hinterlegt wurden. Insgesamt wurden die Pläne mit 13 verschiedenen Software-Herstellern erstellt, wobei bmplife.de am häufigsten verwendet wurde $(n=29)$, gefolgt von medatixx $(n=25)$ und clickdoc.de $(n=10)$. Für 16 BMP konnte die Software nicht bestimmt werden. Im Mittel wurden 7,1 $\pm 3,7$ Präparate pro BMP gezählt ( $\min .=2$, max. $=19$ ), wobei 6,1 $\pm 3,3$ Präparate rezeptpflichtig waren und $1,4 \pm 1,0$ rezeptfrei.

\section{Diskrepanzanalyse}

Von den 100 auf Diskrepanzen untersuchten BMP wiesen 78 \% Abweichungen im Vergleich zur AM-Anamnese auf, mit insgesamt 226 dokumentierten Diskrepanzen (1)-(7) (2,3 \pm 0,6 Abweichungen/Anamnese).

Von 100 untersuchten BMP waren demnach nur 22 BMP inhaltlich identisch zur pharmazeutischen AM-Anamnese (siehe - Abb. 2). Berücksichtigt man außerdem die 177 pharmazeutischen Empfehlungen (E) (betrifft 88 BMP), hätten 6 BMP ohne Kor- rekturen oder pharmazeutische Hinweise als Grundlage für die weitere Therapie im Krankenhaus übernommen werden können. Von den pharmazeutischen Hinweisen und Empfehlungen betrafen die meisten das perioperative Management der Antithrombotika $(n=55)$ und Antidiabetika $(n=28)$, aber auch Empfehlungen zur Dosisanpassung oder bekannte Diagnosen ohne pharmakotherapeutische Behandlung (je $n=18$ ) waren häufig. Hinweise zu unerwünschten Arzneimittelereignissen wurden 10-mal dokumentiert.

Bei der häufigsten Diskrepanz (1) (Arzneimittel fehlt auf dem BMP) waren 60 verschiedene Wirkstoffe betroffen, die sich in 13 der 15 verschiedenen Level-1-ATC-Codes gruppieren ließen. Am häufigsten fehlten Schmerzmittel $(n=14)$, allen voran das rezeptpflichtige Metamizol ( $n=7)$, gefolgt von Antithrombotika $(n=12)$ sowie Mittel gegen Obstipation und Mineralstoffpräparate, die zur Selbstmedikation gezählt werden (je $n=8$, • Abb. 3).

Von den Arzneiformen waren von allen Diskrepanzen erwartungsgemäß orale Arzneiformen wie Tabletten und Kapseln $(n=145)$ am häufigsten betroffen. Von den besonderen Arzneiformen folgten Inhalatoren ( $n=24)$, Fertigspritzen und Arzneiformen zur Anwendung am Auge (je $n=15$ ), wobei 13 der Ophthalmika in die Diskrepanz-Kategorie (1) (Arzneimittel fehlt auf dem BMP) gezählt wurden. Von diesen 13 Ophthalmika enthielten 7 einen Wirkstoff (6 Glaukommittel, 1 Antiphlogistikum), die restlichen Präparate zählten zu den Tränenersatzprodukten, die in der Regel als Medizinprodukt und nicht als Arzneimittel zugelassen sind und damit in die Kategorie Selbstmedikation fallen.

\section{Einfluss von BMP-Eigenschaften auf Art und Anzahl der Diskrepanzen}

In der multivariaten Analyse gab es keinen Zusammenhang zwischen den untersuchten Eigenschaften des BMP und fehlenden (1) oder nicht mehr eingenommenen Arzneimitteln (2). Der Einfluss der verwendeten Software konnte aufgrund der Vielfalt verwendeter Software-Systeme mit zum Teil geringen Stichproben- 


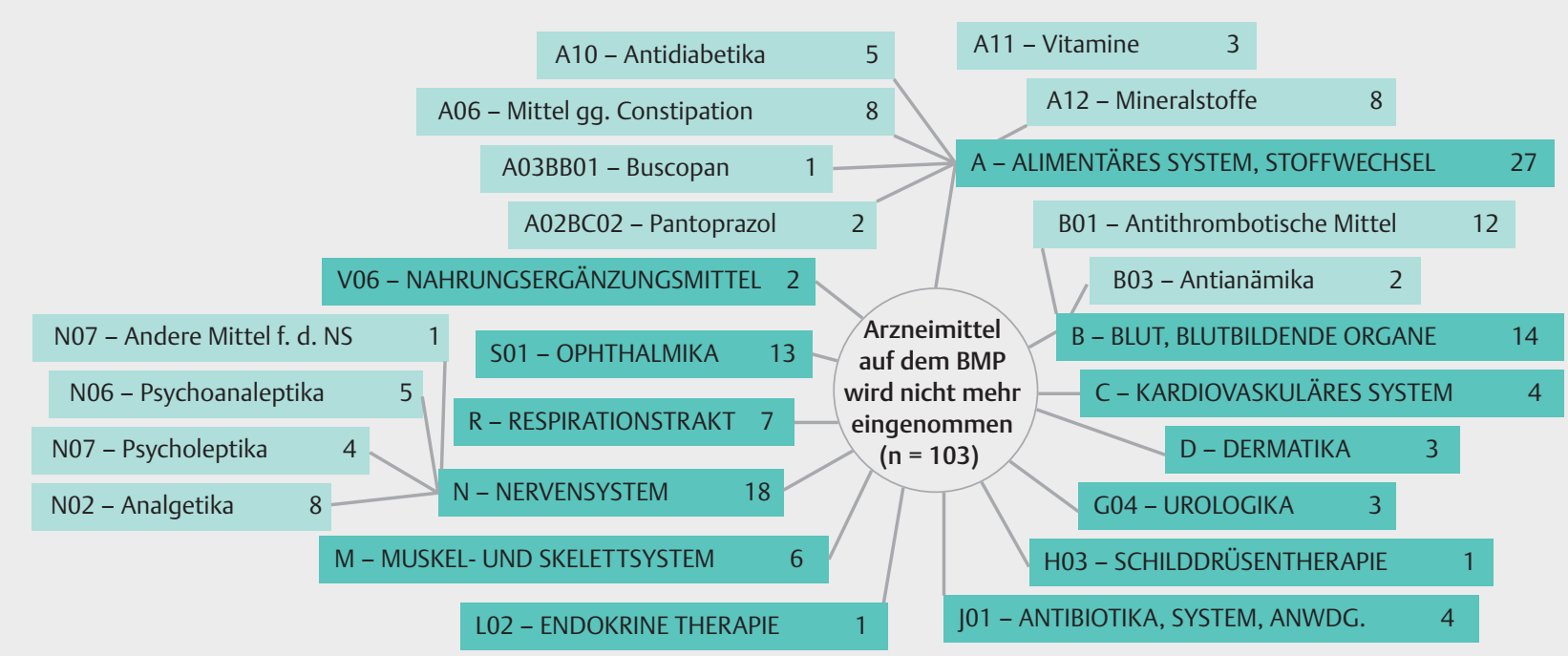

- Abb.3 Auflistung der Arzneimittelgruppen nach ATC-Code und deren Häufigkeit, die im BMP nicht dokumentiert, jedoch laut Patienten eingenommen wurden. Anwdg = Anwendung; f. d. NS = für das Nervensystem; gg = gegen; system = systemische.

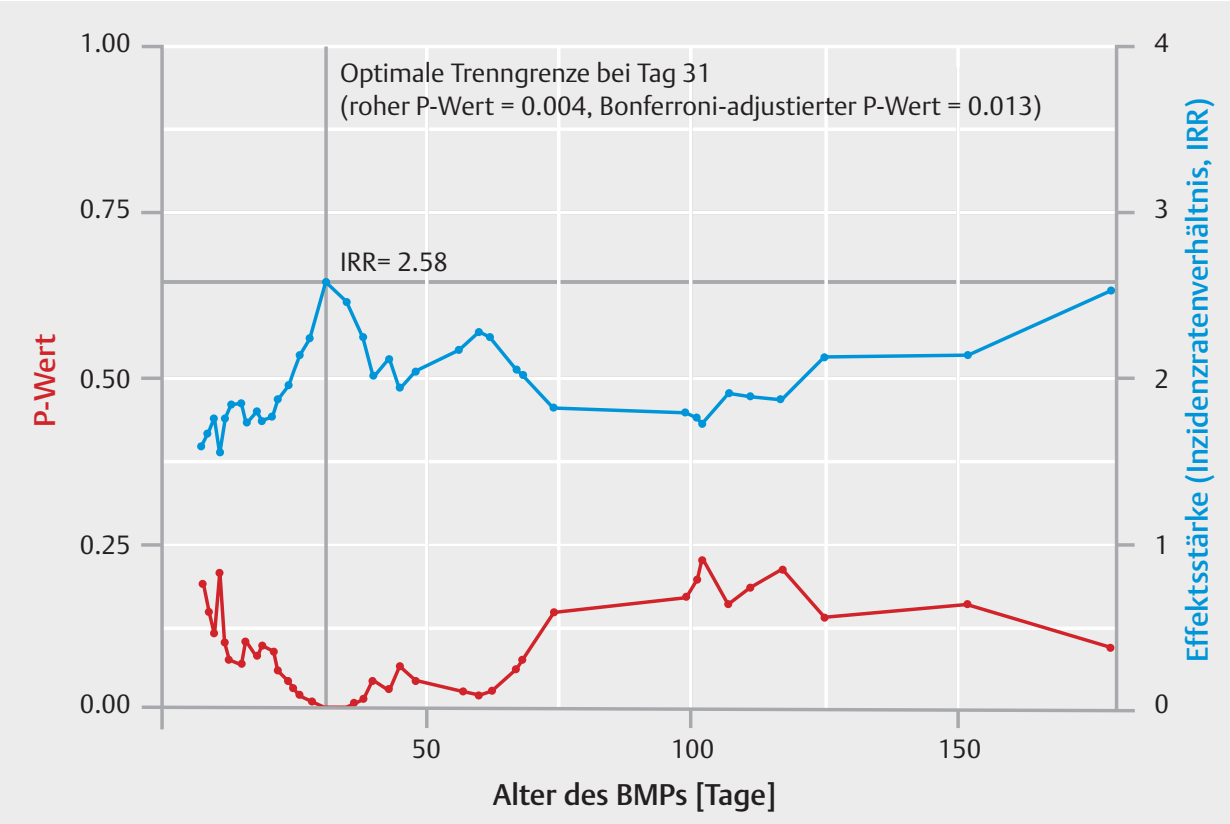

- Abb.4 Optimale Trenngrenze für die Wahrscheinlichkeit von Falschangaben bei Stärke und Dosierung der Arzneimittel in Abhängigkeit des BMP-Alters in Tagen.

größen nicht untersucht werden. Aufgrund der geringen Zahlen für fehlende (3), (4)) und falsche Stärke oder Dosierung (5), (6) wurden diese zu einer Gruppe zusammengefasst. Für fehlende Stärken oder Dosierungen (3), (4)) ergab sich ebenfalls kein statistischer Zusammenhang. Das Risiko für Falschangaben bei Stärke und Dosierung (5), (6) stieg jedoch mit dem Alter des BMP signifikant ( $p=0,047)$. Die Untersuchung zum Einfluss des BMP-Alters für das Auftreten von Falschangaben (5), (6) zeigte ein 2,6-fach erhöhtes Risiko, wenn der BMP älter als 1 Monat war ( $\triangleright$ Abb. 4).

\section{Diskussion}

Die Anzahl mitgebrachter BMP in der AM-Anamnese der Chirurgischen Klinik des Universitätsklinikums Heidelberg war mit 15,3\% aller AM-Anamnesen steigend im Vergleich zu Erhebungen aus den vorherigen Halbjahren (von 3,1 \% im Zeitraum der Einführung 10/2016-03/2017 über 9,5\% zwischen 04/2017 und 09/2017) [10], wobei sich der BMP-Anteil an genutzten Informationsgrundlagen zuletzt (06/2019-01/2020) bei knapp 19\% hielt. Der BMP 
stellt somit eine wichtige, immer häufiger, aber womöglich noch zu selten genutzte Informationsquelle in der AM-Anamnese darselbst wenn bei den knapp $80 \%$ der Patienten ohne BMP einige dabei gewesen sein mögen, die ihren Anspruch auf einen BMP gar nicht eingefordert haben oder aber privat versichert waren und daher formal gar keinen Anspruch gehabt hätten.

Die vorliegende Arbeit zeigt deutlich, dass eine unkritische Übernahme der BMP-Informationen in 78\% der Fälle zu Diskrepanzen mit der tatsächlich aktuell vom Patienten eingenommenen Medikation geführt hätte. Davon betroffen waren vor allem fehlende Angaben (57\%) zu mitunter risikobehafteten Arzneimitteln wie Antithrombotika, Schmerzmitteln und Antidiabetika. Bei den besonderen Arzneiformen waren es vor allem die Inhalativa und Ophthalmika, die auf dem BMP fehlten. Weitere Präparate gehörten zur Selbstmedikation oder waren Nahrungsergänzungsmittel, die ebenfalls wichtig sein können, da sie z. B. Aufschluss geben können über Begleiterkrankungen (z. B. Laxanzien bei Verdauungsstörungen), zu Interaktionen mit der stationären Therapie führen können (z. B. mehrwertige Kationen in Mineralstoffpräparaten mit bestimmten Antibiotika) oder Laborergebnisse beeinflussen können (z. B. Biotin) [11]. Oft fehlten nicht nur Arzneimittel, bei fast einem Drittel standen Arzneimittel auf dem BMP, die laut Patienten nicht mehr eigenommen wurden. Dies deckt sich mit einer Erhebung aus Deutschland von 2015, bei der in öffentlichen Apotheken Medikationspläne auf ähnliche Weise nach Diskrepanzen untersucht wurden: nur 6,5\% der Medikationspläne wiesen keine Diskrepanzen auf, bei $30 \%$ fehlte ein Arzneimittel und $18 \%$ der Pläne verzeichneten Arzneimittel, die bereits nicht mehr eingenommen wurden [12]. Auch in der internationalen Literatur waren auf Medikationsplänen fehlende Arzneimittel die häufigste Diskrepanzart (42-69\%) [4, 5, 8], während die weiteren Diskrepanzkategorien, nicht zuletzt aufgrund unterschiedlicher Methodik und Terminologie, breit streuen. Die hier verwendeten Diskrepranzarten knüpfen an die ausführlichen und klaren Definitionen von Steuerbaut et al. an [8], wobei die vorliegende Arbeit, angepasst an die in Deutschland gegebene Situation der häufig wechselnden Rabattvertragsarzneimittel, die Diskrepanzart „anderer Fertigarzneimittelname“ nicht berücksichtigt. Diskrepanzen scheinen demnach kein spezifisches Problem des BMP zu sein. Dies zeigt aber auch, dass durch die Einführung des BMP bestehende Probleme von Medikationsplänen nicht automatisch gelöst wurden.

Die multivariate Analyse bestätigte den Eindruck aus der täglichen Routine, dass sowohl die aktuellen als auch die älteren Pläne Diskrepanzen zwischen BMP und AM-Anamnese aufwiesen. Nur das Auftreten von Diskrepanzen zu Falschangaben bei Stärke und Dosierung war mit der Aktualität des BMP assoziiert. Für das Anamnesegespräch bleibt es deshalb unerlässlich, jedes auf einem Medikationsplan genannte Präparat mit dem Patienten bzw. seinen Angehörigen zu besprechen und kritische und häufig fehlende Arzneistoffe genauso explizit abzufragen wie besondere Arzneiformen und die Selbstmedikation bzw. Nahrungsergänzungsmittel. Umgekehrt sollte beim Erstellen des BMP auf diese Punkte geachtet werden.

Neben der Überprüfung auf Vollständigkeit der Angaben bzw. der hier gezeigten Diskrepanzanalyse zeigt die Vielzahl pharma- zeutischer Empfehlungen, dass die ABP-Prüfung ein weiterer wichtiger Schritt ist, die Arzneimitteltherapiesicherheit zu erhöhen [13-15]. Gerade zum perioperativen Management oder Erkennen unerwünschter Arzneimittelereignisse können Apotheker einen relevanten Beitrag leisten [16-19]. So wurden und werden weitere Strategien zur interprofessionellen Zusammenarbeit in Studien und Modellprojekten erprobt und validiert, welche die Vollständigkeit und den Nutzen des BMP für Patienten weiter erhöhen sollen [20, 21]. Im Patientengespräch sollte zudem sichergestellt werden, dass die Patienten den BMP auch verstehen und richtig anwenden können. Gerade Ältere und Patienten mit geringerem Bildungsgrad haben Schwierigkeiten bei der Interpretation des BMP [22]. Darüber hinaus bietet es sich an, den Patienten selbst in die Erstellung und Aktualisierung seines Plans einzubinden - sei es über Apps oder andere patientenzentrierte Projekte [23].

Langfristig sollte die Nutzung eines einzigen elektronischen Dokumentes zur Erfassung von Therapieänderungen dazu beitragen, Medikationspläne zumindest halbautomatisch zu aktualisieren und, durch Beteiligung aller involvierten Akteure, die Vollständigkeit der Angaben und damit auch die Arzneimitteltherapiesicherheit zu erhöhen.

\section{KERNAUSSAGEN}

- Der BMP ist eine an den Schnittstellen der Patientenversorgung immer häufiger genutzte Informationsquelle. Eine unkritische Übernahme der Informationen aus dem BMP bei stationärer Aufnahme hätte jedoch in $78 \% \mathrm{zu}$ Abweichungen zur zuletzt tatsächlich vom Patienten eingenommenen Medikation geführt.

- Am häufigsten fehlten auf dem BMP tatsächlich eingenommene Arzneimittel $(n=103)$. Dieser Erhebung nach sollten vor allem Schmerzmittel, besondere Arzneiformen (wie z. B. Augentropfen) und rezeptfreie Arzneimittel gezielt abgefragt und im BMP entsprechend ergänzt werden.

- Neben der Prüfung auf Diskrepanzen zeigen die bei der Anamnese dokumentierten Hinweise und Empfehlungen, dass die Prüfung der Arzneimitteltherapiesicherheit ein wesentlicher Bestandteil des Anamnesegesprächs bleiben sollte.

- Hinsichtlich der untersuchten Eigenschaften der BMP war lediglich das Auftreten von Diskrepanzen zu Falschangaben bei Stärke und Dosierung mit der Aktualität des BMP assoziiert, weshalb in der Praxis Pläne, die älter als einen Monat sind, besonders kritisch hinsichtlich Angaben zu Stärke und Dosierung geprüft werden sollten.

Interessenkonflikt

Die Autorinnen/Autoren geben an, dass kein Interessenkonflikt in Bezug auf die eingereichte Arbeit besteht. Für jeden potenziellen Interessenkonflikt außerhalb der eingereichten Arbeit haben die Autoren die ICMJE-Formulare ausgefüllt. 
Literatur

[1] Tam VC, Knowles SR, Cornish PL et al. Frequency, type and clinical importance of medication history errors at admission to hospital: a systematic review. CMAJ 2005; 173: 510-515. doi:10.1503/cmaj.045311

[2] Cornu P, Steurbaut S, Leysen T et al. Effect of medication reconciliation at hospital admission on medication discrepancies during hospitalization and at discharge for geriatric patients. Ann Pharmacother 2012; 46: 484-494. doi:10.1345/aph.1Q594

[3] Vira T, Colquhoun M, Etchells E. Reconcilable differences: correcting medication errors at hospital admission and discharge. Qual Saf Health Care 2006; 15: 122-126. doi:10.1136/qshc.2005.015347

[4] Cornish PL, Knowles SR, Marchesano R et al. Unintended medication discrepancies at the time of hospital admission. Arch Intern Med 2005; 165: 424-429. doi:10.1001/archinte.165.4.424

[5] Gleason KM, Groszek JM, Sullivan C et al. Reconciliation of discrepancies in medication histories and admission orders of newly hospitalized patients. Am J Health Syst Pharm 2004; 61: 1689-1695. doi:10.1093/ ajhp/61.16.1689

[6] KBV Kassenärztliche Bundesvereinigung. Bundeseinheitlicher Medikationsplan. Im Internet (Stand: 09.01.2020): http://kbv.de/html/ medikationsplan.php

[7] Ärztliches Zentrum für Qualität in der Medizin (ÄZQ) in Zusammenarbeit mit dem Institut für Patientensicherheit und den Projektkrankenhäusern. Leitfaden „Bestmögliche Arzneimittelanamnese“ im Rahmen der High 5s-SOP „Medication Reconciliation“. Im Internet (Stand: 09.01.2020): https://www.aezq.de/mdb/edocs/pdf/patientensicherheit/ leitfaden-arzneimittelanamnese.pdf

[8] Steurbaut S, Leemans L, Leysen T et al. Medication history reconciliation by clinical pharmacists in elderly inpatients admitted from home or a nursing home. Ann Pharmacother 2010; 44: 1596-1603. doi:10.1345/aph.1P192

[9] Altman DG, Lausen B, Sauerbrei W et al. Dangers of using "optimal" cutpoints in the evaluation of prognostic factors. J Natl Cancer Inst 1994; 86: 829-835. doi:10.1093/jnci/86.11.829

[10] Herkert J, Amelung S, Walk-Fritz S et al. Anteil Bundeseinheitlicher Medikationspläne in der Arzneimittelanamnese einer Chirurgischen Klinik. Krankenhauspharmazie 2018; 35: 221-222

[11] ÄrzteZeitung. Biotin kann Laborergebnis verfälschen. In, Rote-HandBrief Neu-Isenburg: Wolfgang van den Bergh. 2019
[12] Waltering I, Schwalbe O, Hempel G. Discrepancies on Medication Plans detected in German Community Pharmacies. J Eval Clin Pract 2015; 21 : 886-892. doi:10.1111/jep. 12395

[13] Kwan JL, Lo L, Sampson M et al. Medication reconciliation during transitions of care as a patient safety strategy: a systematic review. Ann Intern Med 2013; 158: 397-403. doi:10.7326/ 0003-4819-158-5-201303051-00006

[14] Bates DW, Cullen DJ, Laird N et al. Incidence of adverse drug events and potential adverse drug events. Implications for prevention. ADE Prevention Study Group. JAMA 1995; 274: 29-34

[15] Gurwitz JH, Field TS, Harrold LR et al. Incidence and preventability of adverse drug events among older persons in the ambulatory setting. JAMA 2003; 289: 1107-1116

[16] Galvin M, Jago-Byrne MC, Fitzsimons M et al. Clinical pharmacist's contribution to medication reconciliation on admission to hospital in Ireland. Int J Clin Pharm 2013; 35: 14-21. doi:10.1007/s11096-012-9696-1

[17] Mergenhagen KA, Blum SS, Kugler A et al. Pharmacist- versus physicianinitiated admission medication reconciliation: impact on adverse drug events. Am J Geriatr Pharmacother 2012; 10: 242-250. doi:10.1016/ j.amjopharm.2012.06.001

[18] Kwan Y, Fernandes OA, Nagge J] et al. Pharmacist medication assessments in a surgical preadmission clinic. Arch Intern Med 2007; 167: 1034-1040. doi:10.1001/archinte.167.10.1034

[19] Quidley AM, Bland CM, Bookstaver PB et al. Perioperative management of bariatric surgery patients. Am J Health Syst Pharm 2014; 71: 12531264. doi:10.2146/ajhp130674

[20] Korzilius H, Osterloh F. Arzneimitteltherapiesicherheit: Medikationsplan im Praxistest. DtschArztebl 2018; 115: A 364-A 368

[21] Muller U, Schulz M, Matzler M. [Electronically supported co-operation of physicians and pharmacists to improve medication safety in the ambulatory setting: The "Arzneimittelinitiative Sachsen-Thuringen" (ARMIN)]. Bundesgesundheitsblatt Gesundheitsforschung Gesundheitsschutz 2018; 61: 1119-1128. doi:10.1007/s00103-018-2780-5

[22] Botermann L, Monzel K, Krueger K et al. Evaluating patients' comprehensibility of a standardized medication plan. Eur J Clin Pharmacol 2016; 72: 1229-1237. doi:10.1007/s00228-016-2082-5

[23] Universitätsklinikum HD, Abteilungen Allgemeinmed. u. Versorgungsfors./Kli. Pharmakologie u. Pharmakoepidem: Mein Plan. Im Internet (Stand: 09.01.2020): http://www.nimmsrichtig.de/ 